\title{
Diversity-Multiplexing Tradeoff for the Slow Fading Interference Channel
}

\author{
Ekine Akuiyibo \\ Stanford University \\ Packard Electrical Engineering Building \\ 350 Serra Mall \\ Stanford, CA 94305, USA \\ Email: ekine@stanford.edu
}

\author{
Olivier Lévêque \\ Ecole Polytechnique Fédérale de Lausanne \\ Faculté Informatique et Communications \\ Building INR - Station 14 \\ 1015 Lausanne, Switzerland \\ Email: olivier.leveque@epfl.ch
}

\begin{abstract}
We establish the high SNR diversity-multiplexing tradeoff of the fading interference channel, for a general interference level and under the assumption that transmitters and receivers are equipped with a single antenna each.
\end{abstract}

\section{INTRODUCTION}

Considered initially by Shannon in [1], the interference channel describes the shared medium in which two or more transmitter-receiver pairs wishing to communicate reliably, interfere with each other. Although the general capacity region remains unknown in the simplest scenario with two communicating pairs, a recent result by Etkin, Tse and Wang [2] has shed light on the particular case where both interference and noise are additive, and noise is Gaussian. They establish a new outer bound on the capacity region, that is shown moreover to be achievable to within one $\mathrm{bit} / \mathrm{s} / \mathrm{Hz}$ by a simple Han-Kobayashi [3] type scheme, for all values of channel parameters. This characterization of the capacity region is therefore particularly relevant in the high SNR regime, where interference is the main factor limiting communication rates. A refined analysis of this regime is performed in [2], showing that the capacity region highly depends on the interference level considered.

When two transmitter-receiver pairs wish to communicate reliably in a wireless environment, users have to combat channel fading, in addition to dealing with interference. It is of interest to explore the fundamental tradeoffs in this new context. In this paper, we focus on the interferencelimited regime (high SNR), in the scenario where the channel variations over time are sufficiently slow, so that the fading coefficients may be considered as fixed once and for all. If it happens that both transmistters and receivers can get fair estimates of the channel coefficients, then we are back to the situation of the deterministic Gaussian interference channel mentioned above, and Etkin et al. result applies directly; this is summarized in Section II. On the other hand, if only the receivers get estimates of the channel coefficients (as it may happen when feedback links are weak, e.g.), then outage is to be expected. In [4], it has been shown that in the context of point-to-point communications, there is a fundamental tradeoff between the rate at which one intends to communicate and the rate of decrease of the outage probability with SNR (or diversity order). In Section III, we explore this tradeoff in detail in the context of the fading interference channel. The obtained tradeoff highly depends on the interference level considered, just like capacity.

\section{RevieW of EtKin, Tse And WANG's Result}

We consider the fading interference channel

$$
\begin{aligned}
& Y_{1}=h_{11} X_{1}+h_{21} X_{2}+Z_{1} \\
& Y_{2}=h_{12} X_{1}+h_{22} X_{2}+Z_{2}
\end{aligned}
$$

where $Z_{1}, Z_{2}$ are independent noise processes with i.i.d. $\mathcal{N}_{\mathbb{C}}\left(0, N_{0}\right)$ realizations over time and $H=\left(h_{i j}\right)$ is the matrix of channel path gains. We assume that the realizations of $H$ are held fixed over time (slow fading assumption), with the $h_{i j}$ being independent circularly symmetric complex Gaussian random variables with $\operatorname{Var}\left(h_{11}\right)=\operatorname{Var}\left(h_{22}\right)=1$ and $\operatorname{Var}\left(h_{12}\right)=\operatorname{Var}\left(h_{21}\right)=\sigma^{2}$. In addition, the transmitted signals $X_{i} \in \mathbb{C}$ are subject to the power constraint $\mathbb{E}\left\{\left|X_{i}\right|^{2}\right\} \leq P$. For notational convenience, we moreover define

$$
\begin{aligned}
\tilde{X}_{i} & :=\frac{1}{\sqrt{P}} X_{i}, \quad \tilde{Y}_{i}:=\frac{1}{\sqrt{N_{0}}} Y_{i}, \quad \widetilde{Z}_{i}:=\frac{1}{\sqrt{N_{0}}} Z_{i}, \\
\mathrm{SNR} & :=\frac{P}{N_{0}}, \quad \mathrm{INR}:=\frac{P}{N_{0}} \sigma^{2}, \quad g_{i j}:=\frac{h_{i j}}{\sqrt{\operatorname{Var}\left(h_{i j}\right)}} .
\end{aligned}
$$

This leads to the following equivalent representation of the above channel:

$$
\begin{aligned}
& \tilde{Y}_{1}=\sqrt{\mathrm{SNR}} g_{11} \widetilde{X}_{1}+\sqrt{\mathrm{INR}} g_{21} \widetilde{X}_{2}+\widetilde{Z}_{1} \\
& \widetilde{Y}_{2}=\sqrt{\mathrm{INR}} g_{12} \widetilde{X}_{1}+\sqrt{\mathrm{SNR}} g_{22} \widetilde{X}_{2}+\widetilde{Z}_{1}
\end{aligned}
$$

where the normalized channel matrix $G=\left(g_{i j}\right)$ has i.i.d. circularly symmetric complex Gaussian entries with unit variance.

In the scenario where the realizations of $G$ are revealed to both the transmitters and the receivers, the capacity region $\mathcal{C}(G)$ of the above interference channel has been recently characterized by Etkin, Tse and Wang in [2]. More precisely, they show that 
for a given fading matrix $G$, the following rate region is an upper bound on $\mathcal{C}(G)$ :

$$
\begin{aligned}
& \mathcal{R}_{0}(G):=\left\{\left(R_{1}, R_{2}\right) \in \mathbb{R}_{+}^{2}:\right. \\
& \text { a1) } R_{1} \leq \log \left(1+\mathrm{SNR}\left|g_{11}\right|^{2}\right) \\
& \text { a2) } R_{2} \leq \log \left(1+\mathrm{SNR}\left|g_{22}\right|^{2}\right) \\
& \text { b1) } R_{1}+R_{2} \leq \log \left(1+\frac{\mathrm{SNR}\left|g_{11}\right|^{2}}{1+\mathrm{INR}\left|g_{12}\right|^{2}}\right) \\
& +\log \left(1+\operatorname{SNR}\left|g_{22}\right|^{2}+\operatorname{INR}\left|g_{12}\right|^{2}\right) \\
& \text { b2) } R_{1}+R_{2} \leq \log \left(1+\frac{\mathrm{SNR}\left|g_{22}\right|^{2}}{1+\mathrm{INR}\left|g_{21}\right|^{2}}\right) \\
& +\log \left(1+\operatorname{SNR}\left|g_{11}\right|^{2}+\operatorname{INR}\left|g_{21}\right|^{2}\right) \\
& \text { c) } R_{1}+R_{2} \leq \log \left(1+\mathrm{INR}\left|g_{21}\right|^{2}+\frac{\mathrm{SNR}\left|g_{11}\right|^{2}}{1+\mathrm{INR}\left|g_{12}\right|^{2}}\right) \\
& +\log \left(1+\operatorname{INR}\left|g_{12}\right|^{2}+\frac{\operatorname{SNR}\left|g_{22}\right|^{2}}{1+\operatorname{INR}\left|g_{21}\right|^{2}}\right) \\
& \text { d1) } 2 R_{1}+R_{2} \leq \log \left(1+\frac{\mathrm{SNR}\left|g_{11}\right|^{2}}{1+\mathrm{INR}\left|g_{12}\right|^{2}}\right) \\
& +\log \left(1+\operatorname{SNR}\left|g_{11}\right|^{2}+\operatorname{INR}\left|g_{221}\right|^{2}\right) \\
& +\log \left(1+\operatorname{INR}\left|g_{12}\right|^{2}+\frac{\mathrm{SNR}\left|g_{22}\right|^{2}}{1+\mathrm{INR}\left|g_{21}\right|^{2}}\right) \\
& \text { d2) } R_{1}+2 R_{2} \leq \log \left(1+\frac{\mathrm{SNR}\left|g_{22}\right|^{2}}{1+\mathrm{INR}\left|g_{21}\right|^{2}}\right) \\
& +\log \left(1+\mathrm{SNR}\left|g_{22}\right|^{2}+\operatorname{INR}\left|g_{12}\right|^{2}\right) \\
& \left.+\log \left(1+\operatorname{INR}\left|g_{21}\right|^{2}+\frac{\mathrm{SNR}\left|g_{11}\right|^{2}}{1+\mathrm{INR}\left|g_{12}\right|^{2}}\right)\right\}
\end{aligned}
$$

In addition, Etkin, Tse and Wang show in [2] that for any $\left(R_{1}, R_{2}\right) \in \mathcal{R}_{0}(G)$, the rate pair $\left(R_{1}-1, R_{2}-1\right)$ is achievable by a simplification of the Han-Kobayashi scheme, characterizing therefore the capacity region of the Gaussian interference channel to within one bit, for all values of channel parameters.

The high SNR regime is also considered in [2], where the interference level $\alpha$ is defined as the ratio of INR and SNR in $\mathrm{dB}$ :

$$
\alpha:=\frac{\log \mathrm{INR}}{\log \mathrm{SNR}}
$$

Defining further $r_{1}:=\frac{R_{1}}{\log \mathrm{SNR}}$ and $r_{2}:=\frac{R_{2}}{\log \mathrm{SNR}}$, the above upper-bound (1) on the capacity region may be rewritten, in the high SNR limit, as

$$
\begin{aligned}
& r_{1}, r_{2} \leq 1 \\
& r_{1}+r_{2} \leq \max (2-\alpha, \alpha) \\
& r_{1}+r_{2} \leq 2 \max (1-\alpha, \alpha) \\
& \left.\begin{array}{l}
2 r_{1}+r_{2} \\
r_{1}+2 r_{2}
\end{array}\right\} \leq \max (3-2 \alpha, 2,2 \alpha)
\end{aligned}
$$

Since (1) is shown to be achieved to within one bit, this high SNR characterization of the capacity region is tight; $r_{1}$ and $r_{2}$ are referred to in [2] as generalized degrees of freedom.

\section{No Channel KNOWledge AT The TransmitTERS}

Let us now consider the scenario where the channel realizations are revealed to the receivers, but not to the transmitters. More precisely, we assume that receiver 1 gets $\left(\widetilde{Y}_{1}, g_{11}, g_{21}\right)$ and that receiver 2 gets $\left(\widetilde{Y}_{2}, g_{12}, g_{22}\right)$. In the absence of channel knowlegde at the transmitters, the capacity region of the fading interference channel reduces to the single point $\{(0,0)\}$. Indeed, there is always a positive probability for a given non-zero target rate pair $\left(R_{1}, R_{2}\right)$ to fall outside the capacity region. In this section, we analyze this outage probability in detail and establish the high SNR diversitymultiplexing tradeoff for the fading interference channel.

The outage probability is defined as the probability that a required rate pair $\left(R_{1}, R_{2}\right)$ is not in the capacity region of the channel, namely $\mathbb{P}_{\text {out }}\left(R_{1}, R_{2}\right):=\mathbb{P}\left\{\left(R_{1}, R_{2}\right) \notin \mathcal{C}(g)\right\}$. By the above mentioned result [2], we have

$$
\begin{aligned}
\mathbb{P}\left\{\left(R_{1}, R_{2}\right) \notin \mathcal{R}_{0}(g)\right\} & \leq \mathbb{P}_{\text {out }}\left(R_{1}, R_{2}\right) \\
& \leq \mathbb{P}\left\{\left(R_{1}+1, R_{2}+1\right) \notin \mathcal{R}_{0}(g)\right\}
\end{aligned}
$$

Let us therefore define $\mathbb{P}_{\text {out }, 0}\left(R_{1}, R_{2}\right):=\mathbb{P}\left\{\left(R_{1}, R_{2}\right) \notin\right.$ $\left.\mathcal{R}_{0}(g)\right\}$. By the preceding inequality, we see that $\mathbb{P}_{\text {out }}\left(R_{1}, R_{2}\right)$ and $\mathbb{P}_{\text {out }, 0}\left(R_{1}, R_{2}\right)$ behave the same when considering target rates of the order of $\log \mathrm{SNR}$.

For the symmetric rate requirement, we define, with a slight abuse of notation, $\mathbb{P}_{\text {out }}(R):=\mathbb{P}_{\text {out }}(R, R)=\mathbb{P}\{(R, R) \notin$ $\mathcal{C}(G)\}$ and $\mathbb{P}_{\text {out }, 0}(R):=\mathbb{P}_{\text {out }, 0}(R, R)=\mathbb{P}\left\{(R, R) \notin \mathcal{R}_{0}(G)\right\}$. Let us also define

$$
\begin{aligned}
& \mathcal{R}_{0, \mathrm{sym}}(G):=\{R \geq 0: \\
& \text { a) } R \leq \log \left(1+\mathrm{SNR}\left|g_{11}\right|^{2}\right) \\
& \text { b) } 2 R \leq \log \left(1+\frac{\mathrm{SNR}\left|g_{11}\right|^{2}}{1+\mathrm{INR}\left|g_{12}\right|^{2}}\right) \\
&+\log \left(1+\mathrm{SNR}\left|g_{22}\right|^{2}+\mathrm{INR}\left|g_{12}\right|^{2}\right) \\
& \text { c) } 2 R \leq \log \left(1+\mathrm{INR}\left|g_{21}\right|^{2}+\frac{\mathrm{SNR}\left|g_{11}\right|^{2}}{1+\mathrm{INR}\left|g_{12}\right|^{2}}\right) \\
&+\log \left(1+\mathrm{INR}\left|g_{12}\right|^{2}+\frac{\mathrm{SNR}\left|g_{22}\right|^{2}}{1+\mathrm{INR}\left|g_{21}\right|^{2}}\right) \\
& \text { d) } 3 R \leq \log \left(1+\frac{\mathrm{SNR}\left|g_{11}\right|^{2}}{1+\mathrm{INR}\left|g_{12}\right|^{2}}\right) \\
&+\log \left(1+\mathrm{SNR}\left|g_{11}\right|^{2}+\mathrm{INR}\left|g_{21}\right|^{2}\right) \\
&\left.+\log \left(1+\mathrm{INR}\left|g_{12}\right|^{2}+\frac{\mathrm{SNR}\left|g_{22}\right|^{2}}{1+\mathrm{INR}\left|g_{21}\right|^{2}}\right)\right\}
\end{aligned}
$$

By the symmetry of the distribution of the fading coefficients $g_{i j}$, we have

$$
\mathbb{P}_{\text {out }, 0}(R)=\mathbb{P}\left\{R \notin \mathcal{R}_{0, \text { sym }}(G)\right\}
$$

The maximum achievable diversity order for a given target rate pair $\left(R_{1}=r_{1} \log \mathrm{SNR}, R_{2}=r_{2} \log \mathrm{SNR}\right.$ ) (corresponding to 
a multiplexing gain $\left.\left(r_{1}, r_{2}\right)\right)$ is defined as

$$
\begin{aligned}
d\left(\alpha, r_{1}, r_{2}\right) & :=-\lim _{\substack{\mathrm{SNR} \rightarrow \infty \\
\mathrm{NNR}=\mathrm{SNR} \mathrm{R}^{\alpha}}} \frac{\mathbb{P}_{\text {out }}\left(r_{1} \log \mathrm{SNR}, r_{2} \log \mathrm{SNR}\right)}{\log \mathrm{SNR}} \\
& =-\lim _{\substack{\mathrm{SNR} \rightarrow \infty \\
\mathrm{NNR}=\mathrm{SNR} \mathrm{N}^{\alpha}}} \frac{\mathbb{P}_{\text {out }, 0}\left(r_{1} \log \mathrm{SNR}, r_{2} \log \mathrm{SNR}\right)}{\log \mathrm{SNR}}
\end{aligned}
$$

and for the equal rate case, $d_{\text {sym }}(\alpha, r):=d(\alpha, r, r)$. The main result of the paper is given in the following theorem.

Theorem 1. A) For an interference level $\alpha$, the diversity order of the fading interference channel with equal target multiplexing gains $r_{1}=r_{2}=r$ is given by

$$
d_{\text {sym }}(\alpha, r)=\min \left\{d_{a}(\alpha, r), d_{b}(\alpha, r), d_{c}(\alpha, r), d_{d}(\alpha, r)\right\}
$$

where

$$
\begin{aligned}
d_{a}(\alpha, r):= & (1-r)^{+} \forall \alpha \\
d_{b}(\alpha, r):= & 2\left(1-r-\min \left(r, \frac{\alpha}{2}\right)\right)^{+}+(\alpha-2 r)^{+} \\
d_{c}(\alpha, r):= & 2(1-r-\min (r, \alpha))^{+}+2(\alpha-r)^{+} \\
d_{d}(\alpha, r):= & \max \left(\left(1-\frac{3 r}{2}\right)^{+}+(1-3 r)^{+}+2\left(\alpha-\frac{3 r}{2}\right)^{+},\right. \\
& \left.\quad d_{0}(3 r+\min (3 r, 2 \alpha))\right)
\end{aligned}
$$

and $d_{0}(x):=\max (0, \min (3-x, \max (1,2-x)))$

B) In the case of non-equal target multiplexing gains $r_{1}$ and $r_{2}$, the diversity order is given by

$$
\begin{array}{r}
d\left(\alpha, r_{1}, r_{2}\right)=\min \left\{d_{a}\left(\alpha, r_{1}\right), d_{a}\left(\alpha, r_{2}\right), d_{b}\left(\alpha, \frac{r_{1}+r_{2}}{2}\right),\right. \\
\left.d_{c}\left(\alpha, \frac{r_{1}+r_{2}}{2}\right), d_{d}\left(\alpha, \frac{2 r_{1}+r_{2}}{3}\right), d_{d}\left(\alpha, \frac{r_{1}+2 r_{2}}{3}\right)\right\}
\end{array}
$$

Note that the curves $d_{a}(\alpha, r), d_{b}(\alpha, r), d_{c}(\alpha, r), d_{d}(\alpha, r)$ correspond to each bound in (3).

Part A of the theorem is illustrated on Figures 1a and 1b. The function $d(\alpha, r)$ is first represented for three particular values of $\alpha$ on Figure 1a.
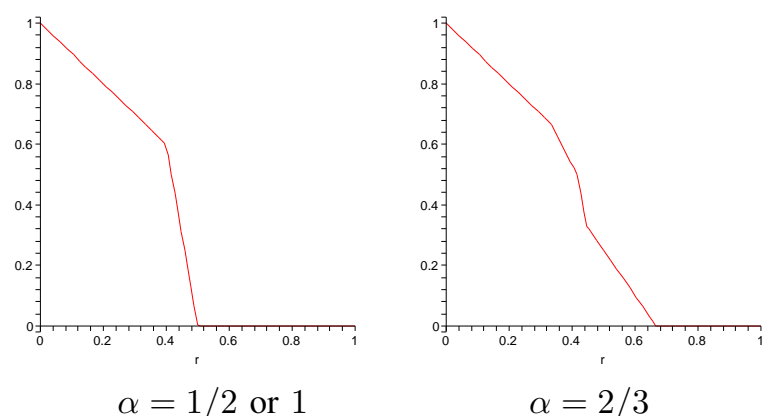

Figure 1a: function $d(\alpha, r)$

The level curves of the function $d(\alpha, r)$ are then represented on Figure 1b. At the bottom of the figure $(r=0), d$ takes uniformly the value 1 for all values of $\alpha$ and decreases more or less rapidly to zero as $r$ increases, depending on the value of $\alpha$. At the top of the figure, one recognizes the w-shaped curve obtained in [2] for the high SNR symmetric capacity, along which the diversity order is zero. On the other hand, the region where only bound a) is effective (i.e. where interference is not limiting diversity) appears also clearly on the figure.

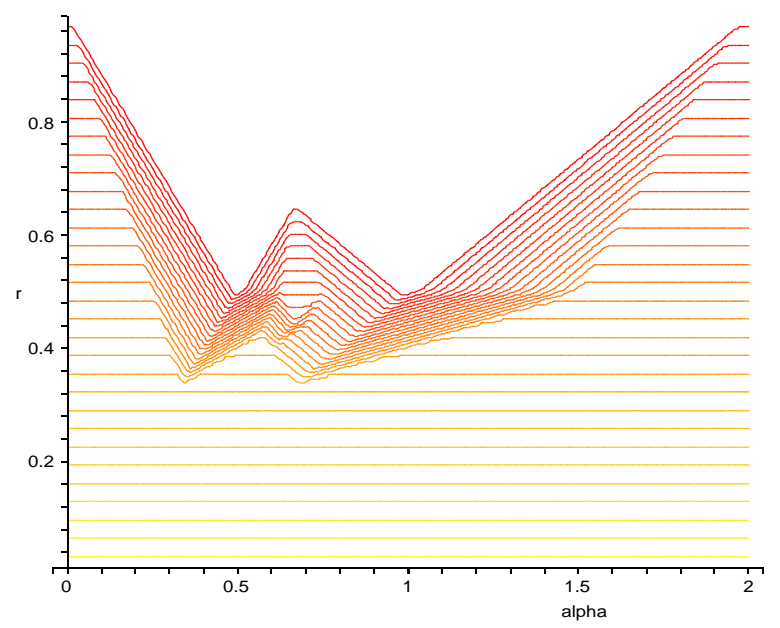

Figure 1b: level curves of $d(\alpha, r)$

Part B of the theorem is illustrated on Figure 2. The level curves of the function $d\left(\alpha, r_{1}, r_{2}\right)$ are represented for different values of $\alpha$. At the lower left corner of the figures $\left(r_{1}=\right.$ $\left.r_{2}=0\right), d$ takes uniformly the value 1 for all values of $\alpha$ and then decreases as $r_{1}, r_{2}$ increase. Depending on the value of $\alpha$, one recognizes the shapes of the high SNR capacity region obtained in [2], along which the diversity order is zero. More interestingly, one can tell from the slope of the level curve which of the bounds among a1, a2, etc. is effective at a given multiplexing gain pair $\left(r_{1}, r_{2}\right)$ (except for the distinction between bounds $\mathrm{b} 1, \mathrm{~b} 2$ and $\mathrm{c})$.

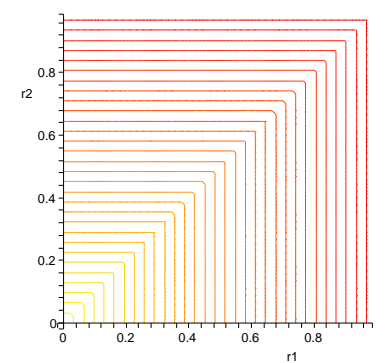

$\alpha=0$ or 2

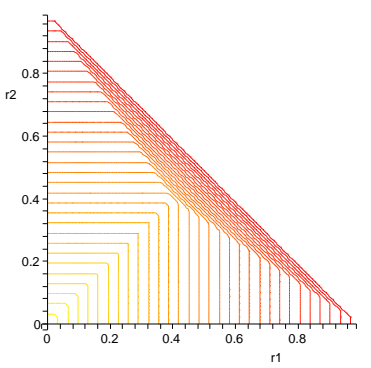

$\alpha=1 / 2$ or 1

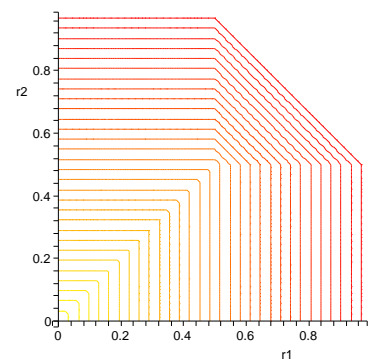

$\alpha=1 / 4$ or $3 / 2$

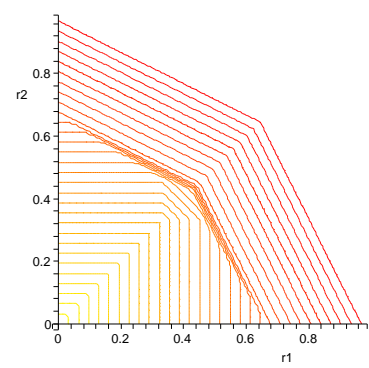

$\alpha=2 / 3$
Figure 2: level curves of $d\left(\alpha, r_{1}, r_{2}\right)$. 
Proof of Theorem 1. A) We follow here the line of analysis developed in [4]. Let $A_{i j}:=\left|g_{i j}\right|^{2}$. Clearly, the $A_{i j}$ are i.i.d. exponential random variables with parameter 1 . The outage probability (4) may then be rewritten as

$\mathbb{P}_{\text {out }, 0}(R)=\int_{\mathcal{D}(A)} e^{-A_{11}-A_{12}-A_{21}-A_{22}} d A_{11} d A_{12} d A_{21} d A_{22}$

where $\mathcal{D}(A):=\left\{A_{i j} \geq 0: R \notin \mathcal{R}_{0, \text { sym }}(A)\right\}$ and $\mathcal{R}_{0, \text { sym }}(A)$ stands for the same interval as that defined in (3), with the random variables $\left|g_{i j}\right|^{2}$ replaced by $A_{i j}$. Let us make further the change of variables $A_{i j}=\mathrm{SNR}^{-\beta_{i j}}$, whose Jacobian is given by $d A_{i j}=(-\log \mathrm{SNR}) \mathrm{SNR}^{-\beta_{i j}} d \beta_{i j}$. This leads to

$$
\mathbb{P}_{\text {out }, 0}(R)=\int_{\mathcal{D}(\beta)} p\left(\beta_{11}, \beta_{12}, \beta_{21}, \beta_{22}\right) d \beta_{11} d \beta_{12} d \beta_{21} d \beta_{22}
$$

with

$$
\begin{gathered}
p\left(\beta_{11}, \beta_{12}, \beta_{21}, \beta_{22}\right)=(\log \mathrm{SNR})^{4} \mathrm{SNR}^{-\beta_{11}-\beta_{12}-\beta_{21}-\beta_{22}} \\
\times e^{-\mathrm{SNR}^{-\beta_{11}}-\mathrm{SNR}^{\beta_{12}}-\mathrm{SNR}^{-\beta_{21}}-\mathrm{SNR}^{-\beta_{22}}}
\end{gathered}
$$

and $\mathcal{D}(\beta)=\left\{\beta_{i j} \in \mathbb{R}: R \notin \mathcal{R}_{0, \mathrm{sym}}(\beta)\right\}$, where again $\mathcal{R}_{0, \text { sym }}(\beta)$ stands for the same interval as that defined in (3) with the $\left|g_{i j}\right|^{2}$ replaced by $\mathrm{SNR}^{-\beta_{i j}}$. Following the analysis and notations ${ }^{1}$ of [4], we further observe that

$$
\begin{aligned}
& \mathbb{P}_{\text {out }, 0}(r \log \mathrm{SNR}) \\
& \quad \doteq \int_{\widetilde{\mathcal{D}}(\beta)} \mathrm{SNR}^{-\beta_{11}-\beta_{12}-\beta_{21}-\beta_{22}} d \beta_{11} d \beta_{12} d \beta_{21} d \beta_{22}
\end{aligned}
$$

where $\widetilde{\mathcal{D}}(\beta):=\left\{\beta_{i j} \geq 0: r \log \operatorname{SNR} \notin \mathcal{R}_{0 \text {,sym }}(\beta)\right\}$. After some ananalysis, and remembering that INR $=\mathrm{SNR}^{\alpha}$, we see that $\widetilde{\mathcal{D}}(\beta)$ may in turn be replaced in the high SNR limit by

$$
\begin{aligned}
& \widehat{\mathcal{D}}(\beta):=\left\{\beta_{11}, \beta_{12}, \beta_{21}, \beta_{22} \geq 0:\right. \\
& \begin{aligned}
a \\
\end{aligned}\left(1-\beta_{11}\right)^{+} \leq r \\
&\text { or } \quad b)\left(\left(1-\beta_{11}\right)^{+}-\left(\alpha-\beta_{12}\right)^{+}\right)^{+} \\
&+\max \left(\left(1-\beta_{22}\right)^{+},\left(\alpha-\beta_{12}\right)^{+}\right) \leq 2 r \\
&\text { or } \quad c) \max \left(\left(\alpha-\beta_{21}\right)^{+},\left(1-\beta_{11}\right)^{+}-\left(\alpha-\beta_{12}\right)^{+}\right) \\
& \quad+\max \left(\left(\alpha-\beta_{12}\right)^{+},\left(1-\beta_{22}\right)^{+}-\left(\alpha-\beta_{21}\right)^{+}\right) \leq 2 r \\
&\text { or } \quad d)\left(\left(1-\beta_{11}\right)^{+}-\left(\alpha-\beta_{12}\right)^{+}\right)^{+} \\
&+\max \left(\left(1-\beta_{11}\right)^{+},\left(\alpha-\beta_{21}\right)^{+}\right) \\
&\left.+\max \left(\left(\alpha-\beta_{12}\right)^{+},\left(1-\beta_{22}\right)^{+}-\left(\alpha-\beta_{21}\right)^{+}\right) \leq 3 r\right\}
\end{aligned}
$$

Using then Laplace's integration method, we obtain

$$
\mathbb{P}_{\text {out }, 0}(r \log \mathrm{SNR}) \doteq \mathrm{SNR}^{-\min _{\widehat{\mathcal{D}}(\beta)} \beta_{11}+\beta_{12}+\beta_{21}+\beta_{22}}
$$

which leads to the following expression for the diversity order:

$$
d_{\text {sym }}(\alpha, r)=\min _{\widehat{\mathcal{D}}(\beta)} \beta_{11}+\beta_{12}+\beta_{21}+\beta_{22}
$$

This optimization problem may be solved separately for the four constraints $a, b, c$ and $d$. Namely,

$$
\begin{aligned}
& d_{\mathrm{sym}}(\alpha, r)=\min \left\{d_{a}(\alpha, r), d_{b}(\alpha, r), d_{c}(\alpha, r), d_{d}(\alpha, r)\right\} \\
& { }^{1} f(\mathrm{SNR}) \doteq g(\mathrm{SNR}) \text { if } \lim _{\mathrm{SNR} \rightarrow \infty} \frac{\log f(\mathrm{SNR})}{\log \mathrm{SNR}}=\lim _{\mathrm{SNR} \rightarrow \infty} \frac{\log g(\mathrm{SNR})}{\log \mathrm{SNR}}
\end{aligned}
$$

where

a) $d_{a}(\alpha, r)=\min \beta_{11} \quad$ subj. to $\left(1-\beta_{11}\right)^{+} \leq r$

b) $d_{b}(\alpha, r)=\min \beta_{11}+\beta_{12}+\beta_{22}$

subj. to $\left(\left(1-\beta_{11}\right)^{+}-\left(\alpha-\beta_{12}\right)^{+}\right)^{+}$

$$
+\max \left(\left(1-\beta_{22}\right)^{+},\left(\alpha-\beta_{12}\right)^{+}\right) \leq 2 r
$$

c) $d_{c}(\alpha, r)=\min \beta_{11}+\beta_{12}+\beta_{21}+\beta_{22}$

subj. to $\max \left(\left(\alpha-\beta_{21}\right)^{+},\left(1-\beta_{11}\right)^{+}-\left(\alpha-\beta_{12}\right)^{+}\right)$

$$
+\max \left(\left(\alpha-\beta_{12}\right)^{+},\left(1-\beta_{22}\right)^{+}-\left(\alpha-\beta_{21}\right)^{+}\right) \leq 2 r
$$

d) $d_{d}(\alpha, r)=\min \beta_{11}+\beta_{12}+\beta_{21}+\beta_{22}$

subj. to $\left(\left(1-\beta_{11}\right)^{+}-\left(\alpha-\beta_{12}\right)^{+}\right)^{+}$

$+\max \left(\left(1-\beta_{11}\right)^{+},\left(\alpha-\beta_{21}\right)^{+}\right)$

$+\max \left(\left(\alpha-\beta_{12}\right)^{+},\left(1-\beta_{22}\right)^{+}-\left(\alpha-\beta_{21}\right)^{+}\right) \leq 3 r$

and we have omitted the constraints $\beta_{i j} \geq 0$ for readability. The solving of these four optimization problems leads to

a) $\beta_{11}^{*}=(1-r)^{+}, \quad$ so $\quad d_{a}(r)=(1-r)^{+}$

b) $\beta_{11}^{*}=\beta_{22}^{*}=\left(1-r-\min \left(r, \frac{\alpha}{2}\right)\right)^{+}, \beta_{12}^{*}=(\alpha-2 r)^{+}$,

so $\quad d_{b}(\alpha, r)=2\left(1-r-\min \left(r, \frac{\alpha}{2}\right)\right)^{+}+(\alpha-2 r)^{+}$

c) $\beta_{11}^{*}=\beta_{22}^{*}=(1-r-\min (r, \alpha))^{+}, \beta_{12}^{*}=\beta_{21}^{*}=(\alpha-r)^{+}$, so $\quad d_{c}(\alpha, r)=2(1-r-\min (r, \alpha))^{+}+2(\alpha-r)^{+}$

d) if $3 r \leq 2 \alpha$, then

$\beta_{12}^{*}=\beta_{21}^{*}=\left(\alpha-\frac{3 r}{2}\right)^{+}, \beta_{11}^{*}=\left(1-\frac{3 r}{2}\right)^{+}, \beta_{22}^{*}=(1-3 r)^{+}$, so $\quad d_{d}(\alpha, r)=\left(1-\frac{3 r}{2}\right)^{+}+(1-3 r)^{+}+2\left(\alpha-\frac{3 r}{2}\right)^{+}$,

if $3 r>2 \alpha$, then

$$
\begin{gathered}
d_{d}(\alpha, r)=\max \left(\left(1-\frac{3 r}{2}\right)^{+}+(1-3 r)^{+}+2\left(\alpha-\frac{3 r}{2}\right)^{+},\right. \\
\left.d_{0}(3 r+\min (3 r, 2 \alpha))\right)
\end{gathered}
$$

which is the result claimed in part A of the theorem ${ }^{2}$.

B) It is straightforward to check that the diversity order for non-equal multiplexing gains $r_{1}$ and $r_{2}$ is obtained by replacing $r$ by $r_{1}$ and $r_{2}$ respectively in bound a; $2 r$ by $r_{1}+r_{2}$ in bounds $\mathrm{b}$ and $\mathrm{c}$; and $3 r$ by $2 r_{1}+r_{2}$ and $r_{1}+2 r_{2}$ respectively in bound $\mathrm{d}$. This completes the proof of the theorem.

\section{ACKNOWLEDGMENT}

We would like to thank Emre Telatar and Christophe Vignat for many helpful discussions.

\section{REFERENCES}

[1] C. E. Shannon, "Two-Way Communication Channels," in Proc. 4th Berkeley Symp. on Mathematical Statistics and Probability, Vol. 1, Berkeley, CA: Univ. California Press, 1961, pp. 611-644.

[2] R. Etkin, D. Tse, H. Wang, "Gaussian Interference Channel Capacity to Within One Bit," submitted to IEEE Trans. Inf. Theory, Feb. 2007.

[3] T. S. Han and K. Kobayashi, "A New Achievable Rate Region for the Interference Channel," IEEE Trans. Inform. Theory, Vol. 27, No 1, pp. 49-60, Jan 1981.

[4] L. Zheng, D. Tse, "Diversity and Multiplexing: A Fundamental Tradeoff in Multiple-Antenna Channels," IEEE Trans. Inform. Theory, vol. 49, May 2003.

${ }^{2}$ Due to space limitations, the derivation of bound $d$ is not made completely explicit here. 